PECEIVEO

CONF-960569--23

MAY 011997

GA-A22359

OSTI

COMPARISON OF WALL/DIVERTOR

DEUTERIUM RETENTION AND PLASMA FUELING

REQUIREMENTS ON THE DIII-D, TdeV, AND

ASDEX-UPGRADE TOKAMAKS

by

R. MAINGI, B. TERREAULT, G. HAAS, G.L. JACKSON, W. ZUZAK, P.K. MIODUSZEWSKI, M.A. MAHDAVI, M.R. WADE, S.C. CHIU, M.J. SCHAFFER, J.T. HOGAN, C.C. KLEPPER

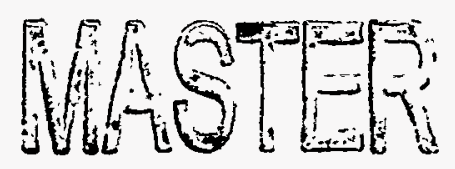

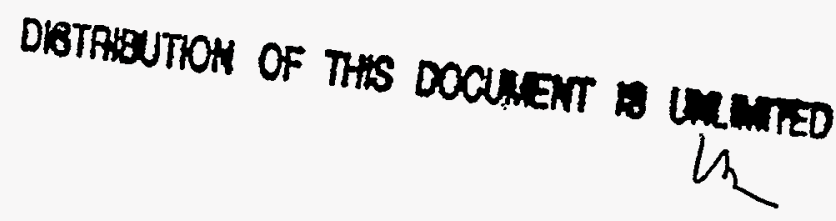

JUNE 1996 


\section{DISCLAIMER}

This report was prepared as an account of work sponsored by an agency of the United States Government. Neither the United States Government nor any agency thereof, nor any of their employees, makes any warranty, express or implied. or assumes any legal liability or responsibility for the accuracy, completeness, or usefulness of any information, apparatus, product, or process disclosed, or represents that its use would not infringe privately owned rights. Reference herein to any specific commercial product, process. or service by trade name, trademark, manufacturer, or otherwise, does not necessarily constitute or imply its endorsement, recommendation, or favoring by the United States Government or any agency thereof. The views and opinions of authors expressed herein do not necessarily state or reflect those of the United States Government or any agency thereof. 


\section{DISCLAIMIER}

Portions of this document may be illegible in electronic image products. Images are produced from the best available original document. 


\title{
COMPARISON OF WALL/DIVERTOR DEUTERIUM RETENTION AND PLASMA FUELING REQUIREMENTS ON THE DIII-D, TdeV, AND ASDEX-UPGRADE TOKAMAKS
}

\author{
by \\ R. MAINGI, ${ }^{*}$ B. TERREAULT, † G. HAAS, $\ddagger$ G.L. JACKSON, W. ZUZAK, $†$ \\ P.K. MIODUSZEWSKI,, M.A. MAHDAVI, M.R. WADE, $\Delta$ S.C. CHIU, \\ M.J. SCHAFFER, J.T. HOGAN, $\Delta$ C.C. KLEPPER ${ }^{\Delta}$
}

This is a preprint of a paper to be presented at the Twelfth International Conference on Plasma Surface Interactions, May 20-24, 1996, Saint Raphael, France, and to be published in The Proceedings.

"Oak Ridge Associated Universities, Oak Ridge, Tennessee.

IInstitut National de la Recherche Scientifique (INRS).

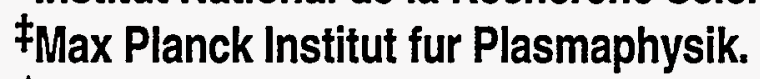

$\Delta_{0}$ Oak Ridge National Laboratory, Oak Ridge, Tennessee.

Work supported by

the U.S. Department of Energy under Contract Nos. DE-AC03-89ER51114 and DE-AC05-84OR21400

GA PROJECT 3466

JUNE 1996 


\section{ABSTRACT}

We present a comparison of the wall deuterium retention and plasma fueling requirements of three diverted tokamaks, DII-D, TdeV, and ASDEX-Upgrade, with different fractions of graphite coverage of stainless steel or Inconel outer walls and different heating modes. Data from particle balance experiments on each tokamak demonstrate well-defined differences in wall retention of deuterium gas, even though all three tokamaks have complete graphite coverage of divertor components and all three are routinely boronized. This paper compares the evolution of the change in wall loading and net fueling efficiency for gas during dedicated experiments without Helium Glow Discharge Cleaning on the DIII-D and TdeV tokamaks. On the DIII-D tokamak, it was demonstrated that the wall loading could be increased by $>1250$ Torr-1 (equivalent to $150 \mathrm{x}$ plasma particle content) plasma inventories resulting in an increase in fueling efficiency from 0.08 to 0.25 , whereas the wall loading on the TdeV tokamak could only be increased by $<35$ Torr- $\ell$ (equivalent to $50 \mathrm{x}$ plasma particle content) plasma inventories at a maximum fueling efficiency $\sim 1$. Data from the ASDEXUpgrade tokamak suggests qualitative behavior of wall retention and fueling efficiency similar to DIII-D. 


\section{INTRODUCTION}

Understanding and characterizing wall retention of hydrogenic species has become an important design issue for next-generation, power-producing, tritium-utilizing devices, such as the International Thermonuclear Experimental Reactor (ITER). Safety considerations will limit the total tritium inventory in such devices. Although extensive research has been done on individual machines $[1,2$, and references therein], multi-machine comparisons are inherently difficult and practically non-existent. This paper compares wall retention and fueling efficiency data from the DIII-D, Tokamak de Varennes (TdeV), and ASDEX-Upgrade tokamaks and suggests the relevant modeling needed to assess quantitatively the differences in observed wall retention.

Control of wall retention during tokamak discharges is accomplished in practice through various wall conditioning techniques, such as baking, boronization, carbonization, helium glow discharge cleaning (HeGDC), Taylor discharge cleaning, disruptive discharge cleaning, beryllium evaporation, lithium pellet injection, electron cyclotron discharge cleaning, etc. [2]. The wall pumping and recycling control that results from such techniques is usually transient, lasting $<60$ $\mathrm{s}$, depending on the fluence to the walls. It has been demonstrated that divertor pumping can be used to unload a loaded wall [3], even in the absence of HeGDC [4]. We have extended the previous study by conducting a similar experiment on $\mathrm{TdeV}$ and also by evaluating fueling characteristics during plasma discharges and deuterium desorption during HeGDC on ASDEXUpgrade. 


\section{DESCRIPTION OF EXPERIMENTS}

\subsection{DIII-D sequence}

The DIII-D tokamak is a medium-sized machine $\left(\mathrm{R}_{0}=1.67 \mathrm{~m}, \mathrm{a}=0.60 \mathrm{~m}\right)$ with $75 \mathrm{~m}^{2}$ internal surface area; approximately $80 \%$ of the internal surfaces and $90 \%$ of the total lower divertor surface area $\left(6 \mathrm{~m}^{2}\right)$ is covered with graphite tiles; the other $10 \%$ in the divertor is boron nitride [3]. Particle control is enabled both by active pumping with three main chamber turbopumps [combined pumping speed $(\mathrm{S}) \sim 7.5 \mathrm{~m}^{3} / \mathrm{s}$ ] and the divertor cryopump ( $\mathrm{S} \sim 25-40 \mathrm{~m}^{3} / \mathrm{s}$, increasing with the plenum pressure) and also passive pumping by wellconditioned graphite tiles. Wall conditioning [5] on DIII-D includes routine, inter-shot HeGDC of 5 minutes duration, boronization, baking, and lithium pellet injection [6]. An experiment was conducted in which the standard HeGDC was disabled, and the net change in wall loading was estimated via a simple global particle balance. The external parameters for that experiment were: $\mathrm{I}_{\mathrm{p}}=1.5 \mathrm{MA}, \mathrm{B}_{\mathrm{t}}=2.0 \mathrm{~T}, \mathrm{P}_{\mathrm{NBI}}=6 \mathrm{MW}, 4.0 \mathrm{~s}$ discharge flat-top in lower single-null diverted configuration. The tokamak was boronized 1 month prior to the execution of this experiment. A detailed account is given in [4] and is summarized below.

A series of 12 , nearly identical discharges was conducted with active pumping provided only by one main chamber turbopump; this sequence was followed by a sequence of 10 discharges in which the divertor cryopump was activated. The net change in wall loading during a discharge cycle $\left(\Delta_{N_{0}}^{\text {wall }}\right)$ is given by a simplified particle balance:

$$
\Delta_{N_{0}}^{w a l l}=\int \Gamma_{0}^{g a s}+\int \Gamma_{0}^{N B I}-\int Q_{0}^{\text {disch }}-\int Q_{0}^{\text {between }}
$$

where $\int \Gamma_{0}^{g a s}$ and $\int \Gamma_{0}^{N B I}$ are the total gas puff and neutral beam inputs during a given discharge, $\int Q_{0}^{\text {disch }}$ is the total cryopump exhaust flux during the discharge, and $\int Q_{0}^{\text {between }}$ is the total exhaust flux (turbopumps + cryopump, when used) between discharges. Note that the plasma content does not enter Eq. (1) as it is zero at the beginning and end of the discharge cycle. Reference discharges with preceding HeGDC (glow voltage $=300 \mathrm{~V}$, glow pressure $\sim 1 \mathrm{mTorr}$, room temperature vacuum vessel wall) were obtained in Phase A, Fig. 1(a). Figure 1(a) displays that without inter-shot HeGDC and without the divertor cryopump (Phase B), the wall loading during plasma discharges far exceeded the exhaust between discharges. With cryopump 

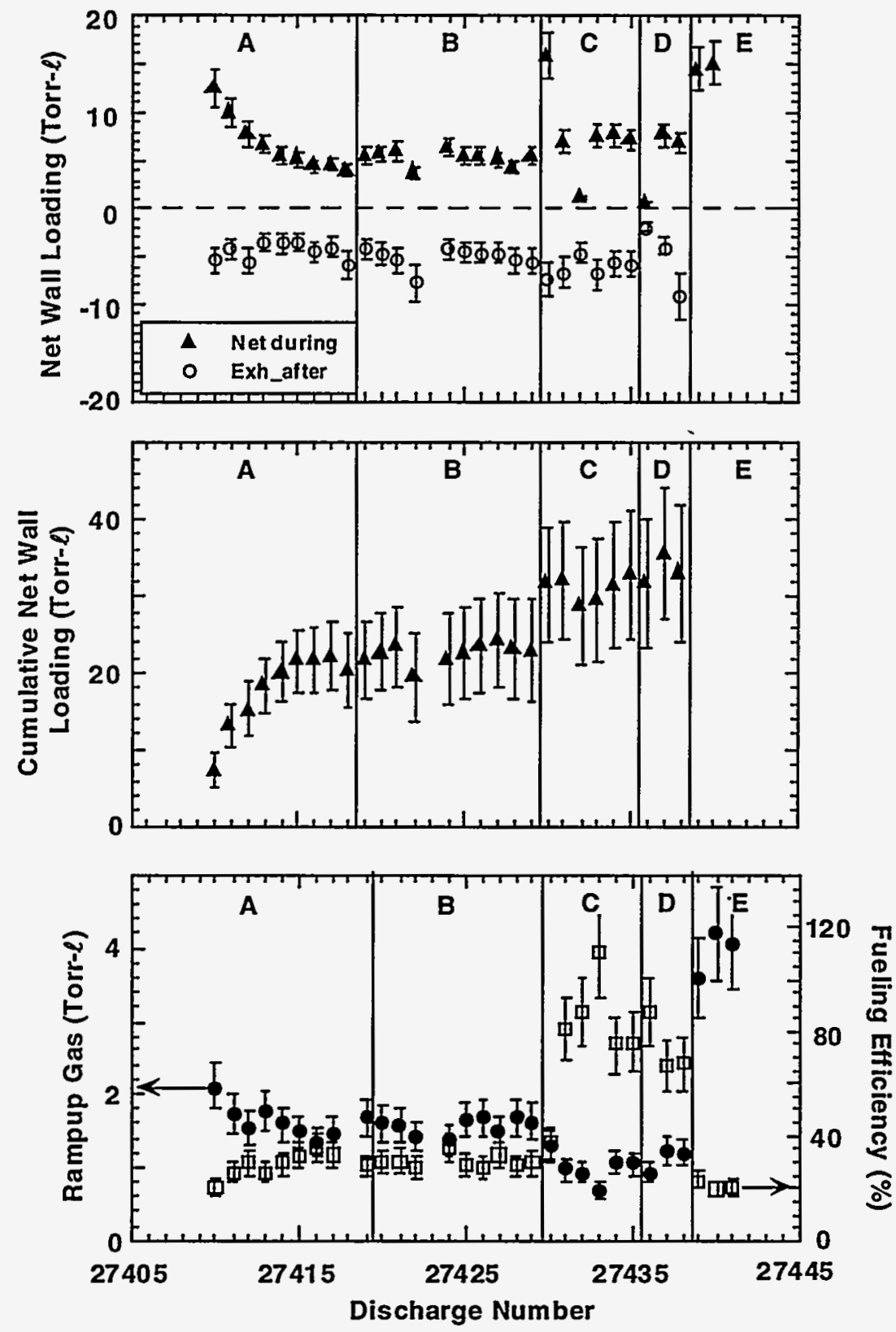

Fig. 1. Evolution of wall loading during sequence without HeGDC for the DIII-D tokamak. (a) Comparison of net wall loading during and after each discharge. Phase A has HeGDC prior to the plasma discharges; Phase $B$ has no HeGDC; and Phase $C$ has an active divertor cryopump. The change in wall loading during discharges is larger the net depletion between discharges in all but the last few discharges. (b) Cumulative change in wall loading vs. discharge number. Two estimates of plenum pressure from the ionization gauge (IG) and capacitance manometer (CM) yield two exhaust rates and thus two wall loading estimates during Phase C. Differences in the measurements and the computed wall loadings are discussed in Ref. [4]. Note that the peak wall loading during Phase $B$ is reduced back to the reference level in Phase $C$. (c) Net cold gas input and fueling efficiency required to achieve the ohmic target density at current flat-top. Discharges 83752-56 were conducted at higher density than preceding discharges. Note the maximum fueling efficiency $\sim 0.25$, indicating that the wall is far from saturated. 
activation after \#83756 (Phase C), the net loading during each discharge was $\leq 0$. Figure 1 (b) demonstrates that the cumulative increase in the wall deuterium retention was 1250 Torr- $\ell$ ( $9 \times$ $10^{22}$ atoms) during Phase $B$; during Phase $C$, this level was reduced back down to the reference level. In comparison, the plasma ohmic inventory at flat-top of the plasma current was $\sim 8$ Torr- $\ell$. A sensitive indicator of the wall retention state is the feedback-controlled external gas input required to reach a prescribed ohmic density in the current ramp-up phase. This rampup gas input is expected to decrease as the wall loading increases. Figure 1(c) shows that this rampup gas, corrected for cryopump exhaust, decreased (increased) strongly as the wall loading was increased (decreased), which resulted in a net fueling efficiency range from 0.08 to 0.25 . Here we define net fueling efficiency as plasma inventory at density flat-top/external gas required for fueling, $\eta_{\text {gas }}=\mathrm{N}_{\text {plasma }}^{\text {flattop }} / \mathrm{N}_{\text {gas }}^{\text {flattop }}$. It is worth noting that our definition of $\eta_{\text {gas }}$ differs from the commonly used fueling/penetration efficiency, defined as $\eta=N_{\text {plasma }} /\left(\tau_{p}^{*} \Gamma_{\text {fuel }}^{\text {gas }}\right)$ where $\Gamma_{\text {fuel }}^{\text {gas }}$ is the instantaneous fueling rate required to maintain a fixed particle content, e.g. in Dylla [7]. The fact that $\eta_{\text {gas }} \ll 1$ suggested that the wall was not yet saturated, implying a wall capacity significantly larger than 1250 Torr- $\ell$.

\subsection{TdeV sequence}

The TdeV tokamak [8] is a small machine $(R=0.87 \mathrm{~m}, \mathrm{a}=0.25 \mathrm{~m})$ with total internal surface area of $34 \mathrm{~m}^{2}$ including two divertor plenums; the top divertor surface area is $0.6 \mathrm{~m}^{2}$. Of these totals, $6 \%$ of the entire non-divertor wall is graphite-covered, and $100 \%$ of the target surface is graphite covered; the remainder is mostly 316 stainless steel. Particle control is enabled both by a main chamber cryopump and turbopumps $\left(\mathrm{S} \sim 4 \mathrm{~m}^{3} / \mathrm{s}\right)$ and divertor cryopumps $\left(\mathrm{S} \sim 6 \mathrm{~m}^{3} / \mathrm{s}\right)$. Wall conditioning includes routine boronization every 1-2 weeks, and HeGDC of variable duration as needed. The external parameters for this experiment were: $I_{p}=0.2 \mathrm{MA}, \mathrm{B}_{t}=1.7 \mathrm{~T}$, $1.0 \mathrm{~s}$ discharge flat-top, lower hybrid rf heating power $\left(\mathrm{P}_{\mathrm{LH}}\right)=400-550 \mathrm{~kW}$, in an upper singlenull diverted configuration. The machine was boronized the day before this experiment. Boronization was followed by a 2 hour HeGDC session (glow voltage $=300 \mathrm{~V}$, glow pressure $\sim 6 \mathrm{mTorr}$, room temperature vessel wall) resulting in an initially depleted wall.

The experiment was conducted in phases with different plasma parameters and active pumping in each phase [Fig. 2(a)]. Phase A is the reference discharge phase with main chamber and divertor pumps active. The divertor cryopumps were de-activated in Phase B; line-average 

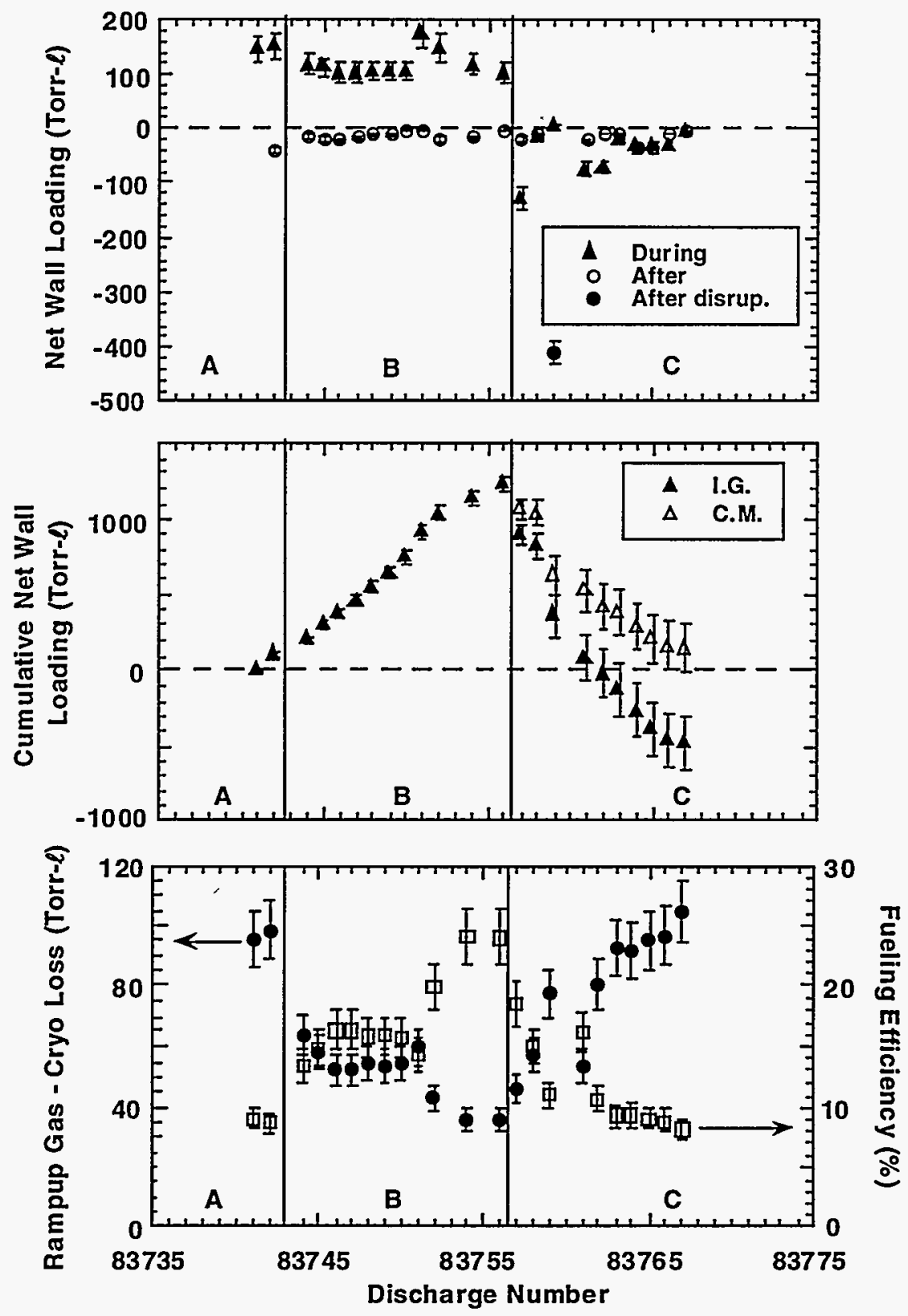

Fig. 2. Evolution of wall loading during sequence without HeGDC for the TdeV tokamak. (a) Comparison of net wall loading during and after each discharge. Phase A: divertor cryopumps on, HeGDC off; Phase B: divertor cryopumps off, HeGDC off, medium $\overline{n_{e}} \sim 5.5 \times 10^{19} \mathrm{~m}^{-3}$; Phase C: high $\overline{n_{e}} \sim 5.5 \times 10^{19} \mathrm{~m}^{-3}$, divertor cryopumps off, HeGDC off; Phase D: divertor cryopumps on, HeGDC off; Phase E: divertor cryopumps off, HeGDC on. After the first 10 discharges, the increase in wall loading during discharges is balanced by the net depletion between discharges. (b) Cumulative change in wall loading vs. discharge number. Note that the phase (27430-437) with $n_{e} \sim 5.5 \times 10^{19} \mathrm{~m}^{-3}$ yields a higher net wall loading than the low density phase. Discharges 27432 and 27436 disrupted shortly after initiation, resulting in low gas fueling. The cumulative particle balance is not computed in Phase E because HEGDC was used. (c) Total cold gas input during rampup and prefill and fueling efficiency required to achieve the ohmic target density at current flat-top. Note the maximum fueling efficiency $\sim 1$, indicating that the wall is almost saturated during the high density phase. 
density $\left(\overline{n_{e}}\right)$ of $3 \times 10^{19} \mathrm{~m}^{-3}$ and $\mathrm{PLH}_{\mathrm{LH}}=400 \mathrm{~kW}$ were used. In Phase $\mathrm{C}$, a higher $\overline{n_{e}}=5.5 \times 10^{19}$ $\mathrm{m}^{-3} \mathrm{~m}^{-3}$ was used in an attempt to alter the wall inventory equilibrium; $\mathrm{P}_{\mathrm{LH}}$ was increased to $550 \mathrm{~kW}$ to prevent radiative collapse at this high density. In Phase $\mathrm{D}$, the divertor cryopumps were re-activated with high $\overline{n_{e}}$ to compare wall loading with the low $\overline{n_{e}}$ in phase A. The divertor cryopumps were de-activated and 3 minutes of $\mathrm{HeGDC}$ was activated in Phase $\mathrm{E}$ for comparison of fueling efficiencies.

The particle balance of Eq. (1) was used to determine the change in wall loading, noting that $\Gamma_{0}^{N B I}$ was 0 and that $\Gamma_{0}^{g a s}$ stayed on for the entire discharge duration. Details of the analysis procedure for the exhaust between discharges is given in [9]. Because of the recent boronization, ten discharges were required to obtain an equilibrium wall retention in Phase A. Figure 2(a) demonstrates that the net loading during plasma discharges was approximately balanced by the exhaust between discharges at the end of Phase A, leading to an increase in the equilibrium wall retention of 20 Torr $\ell$ [Fig. 2(b)]. Figure 2(c) shows that both the rampup gas and fueling efficiency increase gradually to an equilibrium level by the end of Phase $A$ also, indicating static wall conditions. Despite de-activation of the divertor cryopumps in Phase B, the particle balance, wall retention and fueling efficiency were unaffected during discharges \#27420-27429. Even though the pumping speed decreased in this phase, the neutral pressure between discharges increased, leading to comparable total exhaust between discharges. The higher $\overline{n_{e}}$ used in Phase $C$ did lead to a prompt increase in the wall loading to 30 Torr- $\ell$, but this value remained roughly constant over the rest of the discharges in this phase. The fueling efficiency increased more gradually in Phase C, approaching 1 for some discharges; this value of 1 was 4-5 times higher than in the previous phases. Re-activation of the divertor cryopumps in Phase D did not lead to a reduction in wall loading, as observed on DII-D [4]; a high fueling efficiency was maintained in this phase. Finally, activation of $3 \mathrm{~min} H e G D C$ before discharges in Phase $\mathrm{E}$ reduced the fueling efficiency back down to 0.2 , comparable to the values obtained at the beginning of Phase A. The net fueling required to fuel in Phase E discharges increased by $30 \%$ from the discharges in Phase $\mathrm{D}$, suggesting that the HeGDC removed more particles from the wall than the active divertor cryopumps of the preceding discharges.

It is evident from these data that the TdeV tokamak comes into a rapid wall retention equilibrium in the absence of HeGDC. Thus $40 \%$ of the particles injected during the discharge can be accounted for in the divertor cryopump exhaust flux at the end of the discharge, and the remainder can be accounted for in the total exhaust by the main chamber and divertor cryopumps by the beginning of the following discharge [9]. With the divertor cryopumps off, less than 5\% of the particles are exhausted by the main chamber pumps during the discharge, $50 \%$ can be 
accounted for by main chamber cryopump exhaust by $\mathrm{t} \sim 30 \mathrm{~s}$ after the discharge, and the remainder are pumped in the time period from $t=30 \mathrm{~s}$ to just prior to the next discharge.

The peak wall retention in the low density discharges increased by $<25$ Torr $-\ell$, or $\sim 50 \mathrm{x}$ plasma inventory (plasma volume $\sim 1.1 \mathrm{~m}^{3}$ ); during the high density discharges, the peak loading increased to 35 Torr $\ell$, or $\sim 45 \mathrm{x}$ plasma inventory. It is clear that the wall capacity is reached for the high density discharges in Phases $C$ and $D$, where $\eta_{\text {gas }}>0.6$. It should be pointed out that a high $\eta_{\text {gas }}$ is consistent with a low penetration efficiency $(\eta)$ defined in the introduction, in the case that the $\tau_{p}^{*}$ is large, which is expected for a heavily loaded wall. The global results presented here, i.e. good particle accountability and insignificant net change in wall retention, are qualitatively similar to earlier wall retention experiments during the first operational phase of $\mathrm{TdeV}$, which had graphite limiters and stainless steel walls, carbonized by discharge cleaning [10]. These results of low wall retention/robust outgassing are also similar to the results from the ASDEX tokamak with stainless steel walls [11], as well as data from JET with beryllium evaporation [12]. Thus boronization and divertor pumping have a small impact on long-term wall retention of deuterium in TdeV.

\subsection{ASDEX-Upgrade data}

ASDEX-Upgrade is a medium-sized tokamak [13] $\left(\mathrm{R}_{0}=1.625 \mathrm{~m}, \mathrm{a}=0.5 \mathrm{~m}\right)$ with an internal projected area of $72 \mathrm{~m}^{2}$ (flat torus); the surface area for surfaces which are exposed to plasma efflux is somewhat less, $\sim 60 \mathrm{~m}^{2}$. Of the total area, $40 \%$ is graphite covered; the remainder is stainless steel. Active pumping is provided by 14 shielded "divertor" turbo-molecular pumps ( $\mathrm{S}$ $13-18 \mathrm{~m}^{3} / \mathrm{s}$ ). Wall conditioning includes routine, inter-shot HeGDC of 5 minutes duration, boronization, and baking [14]. Because a dedicated experiment similar to the DIII-D and TdeV sequences was not conducted, trends about changes in wall retention are drawn by comparing wall loading during plasma discharges and particle exhaust during succeeding HeGDC sessions at the beginning and end of routine experimental days.

Previous experiments have demonstrated that the $\eta_{\text {gas }} \sim 0.1$ for routine discharges on ASDEX-Upgrade [15], comparable to DII-D. During CDH-mode and other high density, nondisruptive discharges, the net wall loading is increased during the plasma current rampup phase, and it may increase or decrease slightly from this elevated value during the NBI phase $[13,15]$. The HeGDC following plasma discharges removes particles from the wall which were primarily deposited during the preceding plasma discharge. The parameters for standard HeGDC are: glow voltage $=300 \mathrm{~V}$, glow pressure $\sim 1 \mathrm{mTorr}$, and room temperature outer wall. 
As part of an Oak Ridge National Lab/ASDEX-Upgrade collaborative effort, a specially modified Penning gauge was installed near a turbopump on ASDEX-Upgrade. This gauge spectroscopically monitors the deuterium and helium emission in the Penning discharge and can be used to estimate their partial pressures; the implementation is similar to the one developed at TEXTOR [16]. This gauge was used to monitor the species mix during the HeGDC sessions; the deuterium exhaust rate $\left(Q_{d}\right)$ is estimated as the product of the turbomolecular pumping speed and deuterium partial pressure: $\mathrm{Q}_{d}=\mathrm{S}_{\text {turbo }} \mathrm{P}_{\mathrm{d}}$. Integration of $\mathrm{Q}_{\mathrm{d}}$ over the HeGDC duration yields the total deuterium exhaust and associated net reduction in wall loading.

Analysis of data from many routine operational days indicates that the total exhaust during the HeGDC process is lowest at the beginning of the run day, and increases by up to $2 \mathrm{x}$ at the end of the run day for a fixed HeGDC duration of 5 minutes. Figure 3 displays the deuterium partial pressure during a HeGDC near the beginning of a run day, preceding discharge \#6279; the total exhaust at the end of the HeGDC is $\sim 80$ Torr- $\ell$. The pressure decay can be fit with a double exponential with fast and slow time constants of $36 \mathrm{~s}$ and $159 \mathrm{~s}$ respectively; by extrapolation, an infinitely long HeGDC would have removed $\sim 115$ Torr $-\ell$. The pressure decay rate was about the same during one of the last HeGDC sessions of the same run day, preceding discharge \#6294; the total deuterium removal during the HeGDC was $\sim 110$ Torr- $\ell$. Fitting the pressure decay with a double exponential yielded fast and slow time constants of $27 \mathrm{~s}$ and $180 \mathrm{~s}$ respectively, with an extrapolated removal of 130 Torr $-\ell$ for an infinitely long HeGDC. Thus more particles were desorbed with the same HeGDC duration at the end of the run day than the beginning of the run day, suggesting that the wall loading actually increased during the run day. In comparison, the net wall loading during discharge \#6278 and \#6293 using Eq. (1) was $\sim 160$ Torr- $\ell$ and 130 Torr- $\ell$ respectively, indicating net wall loading $\leq 80$ Torr- $\ell$ for the shot sequence at the beginning of the day and $\leq 20$ Torr $\ell$ for the sequence at the end of the day. This wall evolution is not unique to the day that the above data was analyzed. Analysis of data from the previous run day indicates a HeGDC D 2 removal of 60 Torr $\ell$ preceding the $6^{\text {th }}$ discharge and 110 Torr- $\ell$ preceding the $20^{\text {th }}$ discharge; also, the net wall loading during the 5 th and 19th discharges each exceeded the amount of the following HeGDC session. The discharges discussed here were similar in plasma parameters and did not directly follow a disruption. Thus the wall naturally "unloads" in the period between run days (two days for the above data, no external wall conditioning applied other than a HeGDC prior to operations). These inferences are supported by the fact that the tokamak base neutral pressure typically rises during the course of a run day, and also by the fact that more gas pre-fill before discharge initiation is required at the beginning of a run day as compared with the end of the day. Both of these quantities return to nominal values by the beginning of the following run day [14]. 


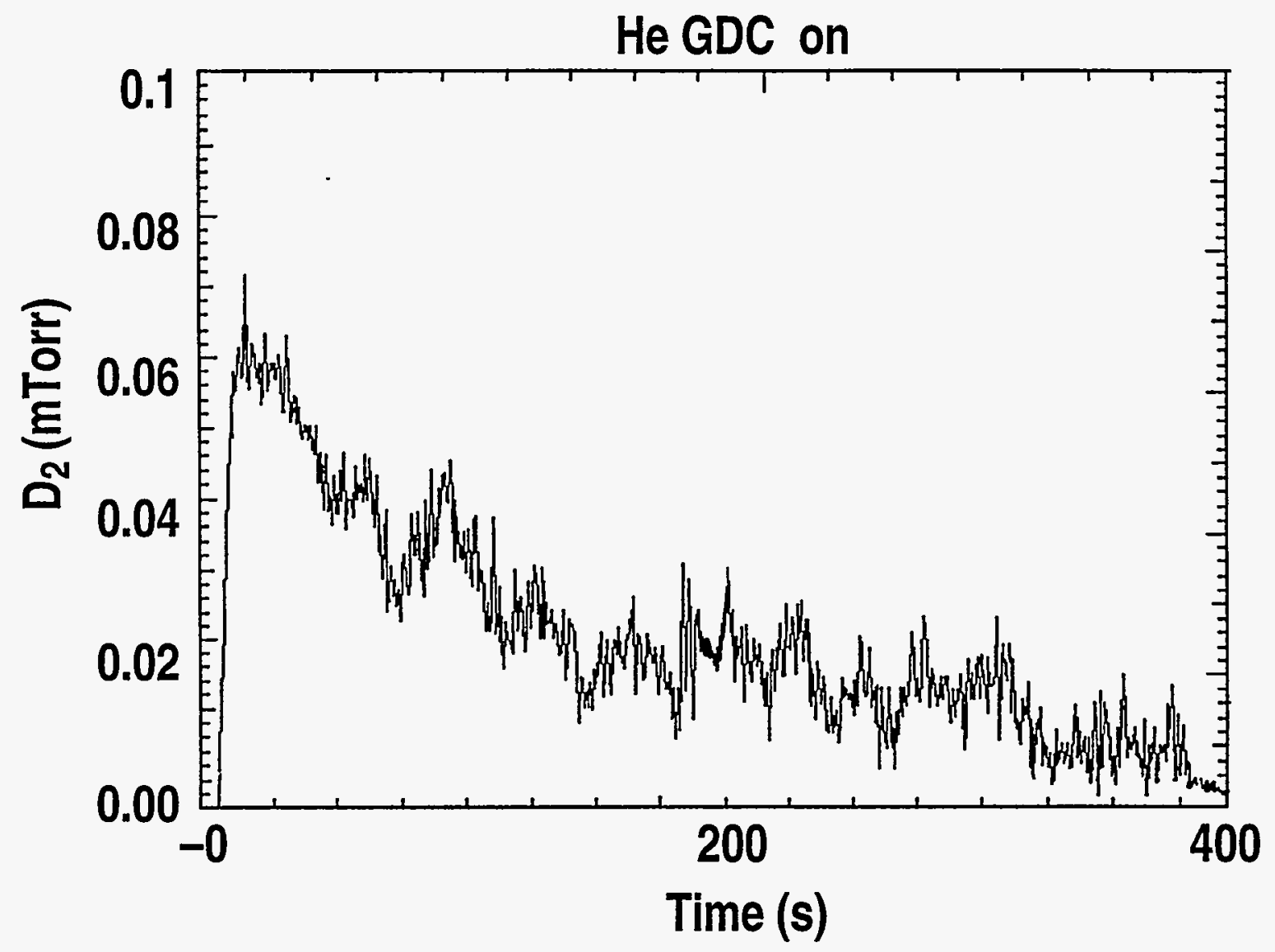

Fig. 3. Decay of deuterium partial pressure in ASDEX-Upgrade during HeGDC after one of the first discharges of a run day (preceding discharge \#6279). 


\section{DISCUSSION}

It has been demonstrated that the wall capacity on DIII-D is well in excess of 150 typical plasma inventories. The behavior of wall retention on ASDEX-Upgrade appears to be qualitatively similar to DIII-D in that wall loading typically evolves over a long time-scale. In addition, both the DIII-D and ASDEX-Upgrade have a cold gas fueling efficiency $\sim 0.1$. In contrast, wall retention in $\mathrm{TdeV}$ can be increased $<50$ plasma inventories. Dividing the wall retention by the total wall surface area yields $1.2 \times 10^{21} \mathrm{atom} / \mathrm{m}^{2}$ and $8.2 \times 10^{19} \mathrm{atom} / \mathrm{m}^{2}$ for DIII-D and TdeV respectively. On the other hand, dividing by the total graphite surface area in each tokamak yields about $1.5 \times 10^{21}$ atom $/ \mathrm{m}^{2}$ for both tokamaks. Assuming a graphite density of $2.25 \mathrm{~g} / \mathrm{cm}^{3}$ and a surface penetration depth $\sim 0.5 \AA / \mathrm{eV}$, the required average energy of incident flux to populate the required wall surface depth for both tokamaks is $\sim 300 \mathrm{eV}$. These energies are reasonable only for charge exchange neutral outflux from the core plasma, which must contribute to long-term wall retention significantly more than colder ions near the divertor strike points. From these arguments, it is likely that the presence of non-divertor graphite allows a high wall loading relative to divertor graphite surface area on DIII-D, and the lack of extensive nondivertor graphite coverage in $\mathrm{TdeV}$ limits its maximum wall retention.

Because the characteristics of particle balance have not changed on $\mathrm{TdeV}$ from the first operational phase (limiter and limited wall conditioning) to the current one (divertor, extensive wall conditioning), we hypothesize that the wall conditioning does not affect the long-term deuterium retention in TdeV. Significant differences in DII-D and TdeV include the heating mechanism, NBI vs. rf, and the non-divertor surface composition. NBI leads to a population of energetic charge-exchange neutrals which can penetrate deep into wall surfaces. Recent modeling of the DIII-D experiment suggests that high energy charge exchange neutrals in the footprint of the divertor strike points and also the outer wall can lead to long-term retention in the graphite tiles [17]. Modeling of the TdeV data with edge plasma and neutral transport codes should provide insight into the relative importance of heating mechanism and wall composition on wall retention. This modeling may also provide insight into why the boronized TdeV tokamak has the retention and outgassing characteristics of an unboronized metal wall machine. 


\section{REFERENCES}

[1] J. Ehrenberg, J. Nucl. Mater. 162-164 (1989) 63.

[2] J. Winter, Plasma Phys. Contr. Fusion 36 (1994) B263.

[3] M.A. Mahdavi, et al., Proc. 20th Euro. Conf. on Contr. Fusion, Lisbon, Portugal, Vol. $17 \mathrm{C}$ (1993) II-747.

[4] R. Maingi, et. al., Nucl. Fusion 36 (1996) 245.

[5] G.L. Jackson and the DIII-D Team, J. Vac. Sci. Technol. A 10 (1992) 1244.

[6] G.L. Jackson, these proceedings.

[7] H.F. Dylla, et. al., J. Nucl. Mater. 111-112 (1982) 211.

[8] R. Decoste, et. al., Proc. 18th Euro. Conf. on Contr. Fusion, Berlin, Germany, Vol. 15C (1991) I-141.

[9] R. Maingi, et. al., TdeV Note Technique 95-010, July 1995 (unpublished)

[10] B. Terreault, et al., J. Nucl. Mater. 176-177 (1990) 422.

[11] Y.G. Wang, et. al., J. Vac. Sci. Techn. A4 (1986) 2520.

[12] J. Ehrenberg, et. al., J. Nucl. Mater. 196-198 (1992) 992.

[13] K. Lackner, et al., Plasma Phys. Contr. Fusion 36 (1994) B79.

[14] G. Haas, private communication, Max Planck Institut für Plasmaphysik, Garching, 1996.

[15] G. Haas, et. al., Proc. 22nd Euro. Conf. on Contr. Fusion, Bournemouth, U.K., Vol. 19C (1995) I-321.

[16] K. H. Finken, et. al., Rev. Sci. Instrum. 63 (1992) 1.

[17] J. Hogan, et. al., these proceedings. 


\section{ACKNOWLEDGMENTS}

This work was supported by the U.S. Department of Energy under Contract Nos. DE-AC03-89ER51114 and DE-AC05-84OR21400. The efforts of the DIII-D, TdeV, and ASDEX-Upgrade operations staff are gratefully acknowledged. The CCFM is funded by AECL, Hydro-Quebec, and INRS. The first author is funded by a Oak Ridge National Laboratory Postdoctoral Fellowship, administered by Oak Ridge Institute for Science and Education. 